REVESCO. Revista de Estudios Cooperativos

ISSN: $1885-8031$

http://dx.doi.org/10.5209/REVE.59773

\title{
Sociedades de responsabilidad limitada calificables y sociedades participadas: una aproximación a su cuantificación
}

\author{
Paloma Bel Durán ${ }^{1}$ y Gustavo Lejarriaga Pérez de las Vacas ${ }^{2}$
}

Recibido: 25 de enero de 2018 / Aceptado: 5 de febrero de 2018

Resumen. El presente artículo se basa en el proyecto de investigación de título "Estudio del concepto de sociedad participada a partir de las sociedades laborales no calificadas" realizado conjuntamente entre la Escuela de Estudios Cooperativos de la Facultad de Ciencias Económicas y Empresariales de la Universidad Complutense de Madrid y la Confederación de Sociedades Laborales (CONFESAL) al amparo la Orden ESS/1338/2013 del Ministerio de Empleo y Seguridad Social (Dirección General del Trabajo Autónomo, de la Economía Social y de la Responsabilidad Social de las Empresas) en su convocatoria del año 2016 .

El objetivo básico del proyecto, cuyos resultados se reflejan en este artículo, es ofrecer una aproximación cuantitativa de las sociedades participadas que hay en España a partir del concepto de sociedad laboral "no calificada", partiendo de un trabajo de campo realizado en gran parte del territorio español sobre sociedades de responsabilidad limitada activas y de nueva creación.

Palabras clave: Sociedad de responsabilidad limitada; Sociedad laboral; Sociedad participada; Cuantificación.

Claves Econlit: P19; L260; J540; M100.

\section{[en] Limited qualified liability companies and participated companies: an approximation to their quantification}

\begin{abstract}
This article is based on the research project "Study of the participated companies from labor companies not qualified" realize by the School of Cooperative Studies of the Faculty of Economic and Business Sciences of the Complutense University of Madrid and the Confederation of Labor Societies (CONFESAL) under the Order ESS / 1338/2013 of the Ministry of Employment and Social Security (General Directorate of Autonomous Work, Social Economy and Social Responsibility of Companies).

The basic objective of the project whose results are reflected in this article is to offer a quantitative approximation of the participated companies that exist in Spain.

Keywords: Limited liability company; Labor company; Participated company; Quantification.
\end{abstract}

Sumario. 1. Introducción. 2. Aportaciones de la Ley 44/2015, de 14 de octubre, de Sociedades Laborales y Participadas. 3. Sociedad laboral calificada frente a sociedad laboral calificable. 4. Una

1 Universidad Complutense de Madrid, España

Dirección de correo electrónico: pbeldura@ucm.es.

2 Universidad Complutense de Madrid, España

Dirección de correo electrónico: grlejarr@ucm.es. 
aproximación cuantitativa de las sociedades participadas. 5. Resultados. 6. Conclusiones. 7. Referencias bibliográficas.

Cómo citar: Bel Durán, P. y Lejarriaga Pérez de las Vacas, G. (2018) Sociedades de responsabilidad limitada calificables y sociedades participadas: una aproximación a su cuantificación. REVESCO. Revista de Estudios Cooperativos, Primer Cuatrimestre, $\mathrm{N}^{\circ}$ 127, pp. 9-25. DOI: 10.5209/REVE.59773.

\section{Introducción}

En todo el periodo de crisis económica en España entre los años 2008 y 2013, la única forma empresarial que no ha visto reducido en términos porcentuales su número de entidades con actividad por encima de los dos dígitos han sido las sociedades de responsabilidad limitada. Como se aprecia en la Tabla 1, la pérdida de empresas bajo esta forma jurídica en el periodo referido se ha situado en un 1,78 por ciento, cifra muy por debajo de la media del conjunto $(-8,06$ por ciento) y del resto de fórmulas habituales (sociedades anónimas y sociedades cooperativas, con descensos superiores al 14 por ciento, y empresas individuales, con valores cercanos al 13 por ciento).

Tabla. 1. Variación relativa de empresas activas en el periodo 2008-2013 por formas jurídicas

\begin{tabular}{llcclc}
\hline & $\begin{array}{l}\text { Sociedades } \\
\text { Anónimas }\end{array}$ & $\begin{array}{l}\text { Sociedades de } \\
\text { Responsabilidad } \\
\text { Limitada }\end{array}$ & $\begin{array}{l}\text { Sociedades } \\
\text { Cooperativas }\end{array}$ & $\begin{array}{l}\text { Personas } \\
\text { Físicas }\end{array}$ & Total \\
\hline España & $-14,61 \%$ & $-1,78 \%$ & $-14,38 \%$ & $-12,83 \%$ & $-8,06 \%$ \\
\hline
\end{tabular}

Fuente: Elaboración propia a partir del Directorio Central de Empresas (DIRCE) del Instituto Nacional de Estadística (INE):

http://www.ine.es/dyngs/INEbase/es/operacion.htm?c=Estadistica_C\&cid=1254736160707 $\&$ menu $=u$ ltiDatos\&idp=1254735576550. Fecha de consulta: 23 de enero de 2018.

Los síntomas de recuperación en el trienio 2013-2016 son evidentes (Tabla 2) al observar el crecimiento de empresas activas en su conjunto por el comportamiento tanto de las empresas individuales (crecen casi un 4 por ciento, pero hay que tener en cuenta que su descenso en el periodo precedente fue muy pronunciado) como de las sociedades de responsabilidad limitada (que aumentan en casi un 2,5 por ciento habiendo demostrado su resistencia durante los años 2008-2013). 
Tabla 2. Variación relativa de empresas activas en el periodo 2013-2016 por formas jurídicas

\begin{tabular}{lccccc}
\hline & $\begin{array}{l}\text { Sociedades } \\
\text { Anónimas }\end{array}$ & $\begin{array}{l}\text { Sociedades de } \\
\text { Responsabilidad } \\
\text { Limitada }\end{array}$ & $\begin{array}{l}\text { Sociedades } \\
\text { Cooperativas }\end{array}$ & $\begin{array}{l}\text { Personas } \\
\text { Físicas }\end{array}$ & Total \\
\hline España & $-11,56 \%$ & $2,47 \%$ & $-2,00 \%$ & $3,84 \%$ & $2,86 \%$ \\
\hline
\end{tabular}

Fuente: Elaboración propia a partir del Directorio Central de Empresas (DIRCE) del Instituto Nacional de Estadística (INE):

http://www.ine.es/dyngs/INEbase/es/operacion.htm?c=Estadistica_C\&cid=1254736160707

$\&$ menu $=$ ultiDatos\&idp=1254735576550. Fecha de consulta: 23 de enero de 2017.

Frente a ello, las sociedades de responsabilidad limitada laborales (al igual que las sociedades anónimas laborales) no han mantenido esta tendencia. Los datos del Ministerio de Empleo sobre las sociedades laborales en España en el periodo 20082016, indican una tendencia decreciente, sin síntoma alguno de recuperación.

Las variaciones relativas referidas más que demuestran un comportamiento muy aceptable de las sociedades de responsabilidad limitada activas en todo el periodo de análisis considerado no son consecuencia de que la mortandad de este tipo de empresas haya sido baja, que no lo ha sido, sino de que han seguido creándose empresas a un ritmo nada despreciable.

$\mathrm{Y}$ aquí es donde hay una diferencia muy importante con relación a las sociedades laborales (también la hay en mortandades o pérdidas de condición laboral, pero no tan acusada).

En efecto, en el año 2007, se registraron en España 2.324 sociedades laborales, mientras que en el año 2016 solo se dieron de alta en registro 414 empresas (1.910 menos); esto es, un 82 por ciento menos.

Según datos del Directorio Central de Empresas del Instituto Nacional de Estadística, en 2007 se dieron de alta 129.028 sociedades de responsabilidad limitada. En 2016 las altas se situaron en 99.590 entidades. Evidentemente, el ritmo de creación de nuevas empresas se ralentizó entre estos años. Entre 2007 y 2016 la diferencia son 29.440 sociedades de responsabilidad limitada menos constituidas lo que pone de manifiesto una variación relativa de casi un 23 por ciento. Con todo, se evidencia, una reducción mucho más dramática en el caso de las sociedades laborales.

Como puede apreciarse en la Tabla 3, el ritmo de creación de sociedades de responsabilidad limitada laborales se ha reducido en un 82 por ciento frente al 23 por ciento de su fórmula jurídica de base. Evidentemente, se ha perdido gran parte del interés existente en adquirir la condición laboral. 
Tabla. 3. Número de Sociedades de Responsabilidad Limitada Laborales y no Laborales registradas en España en el periodo 2008-2015

\begin{tabular}{lccccccccccc}
\hline & $\mathbf{2 0 0 7}$ & $\mathbf{2 0 0 8}$ & $\mathbf{2 0 0 9}$ & $\mathbf{2 0 1 0}$ & $\mathbf{2 0 1 1}$ & $\mathbf{2 0 1 2}$ & $\mathbf{2 0 1 3}$ & $\mathbf{2 0 1 4}$ & $\mathbf{2 0 1 5}$ & $\mathbf{2 0 1 6}$ & $\begin{array}{l}\text { Total } \\
\text { 2007-2016 } \\
\text { Variación } \\
\text { relativa }\end{array}$ \\
\hline SLL & 2.324 & 1.488 & 1.204 & 1.236 & 1.130 & 986 & 877 & 756 & 506 & 414 & $-82 \%$ \\
$\begin{array}{l}\text { SL } \\
\text { (miles) }\end{array}$ & 129,03 & 98,18 & 83,01 & 81,87 & 82,44 & 83,90 & 92,11 & 91,70 & 90,46 & 99,59 & $-23, \%$ \\
\hline
\end{tabular}

Fuente: Elaboración propia a partir de la Base de Datos de la Economía Social del

Ministerio de Empleo y Seguridad Social. Disponible en Internet:

http://www.empleo.gob.es/es/sec_Trabajo/autonomos/economia-

soc/EconomiaSocial/estadisticas/ y Directorio Central de Empresas del Instituto Nacional de Estadística (DIRCE):

http://www.ine.es/jaxi/menu.do?type $=$ pcaxis $\&$ path $=\% 2 \mathrm{Ft} 37 \% 2 \mathrm{Fp} 201 \&$ file $=$ inebase $\& \mathrm{~L}=0$

Fecha de consulta: 22 de enero de 2018.

Con respecto a las sociedades de responsabilidad limitada laborales activas, se observa una drástica disminución tanto en sociedades como en trabajadores. También se observa una disminución en su dimensión, comportamiento contrario en el caso de las sociedades de responsabilidad limitada laborales.

Tabla. 4. Sociedades de Responsabilidad Limitada Laborales activas en el periodo 2008-2015

\begin{tabular}{|c|c|c|c|c|c|c|}
\hline Años & 2008 & & & 2016 & & \\
\hline & Sociedades & Trabajadores & $\begin{array}{l}\text { Dimensión } \\
\text { media }\end{array}$ & Sociedades & Trabajadores & $\begin{array}{l}\text { Dimensión } \\
\text { media }\end{array}$ \\
\hline $\begin{array}{l}\text { Sociedades de } \\
\text { responsabilidad } \\
\text { limitada } \\
\text { laborales }\end{array}$ & 15.480 & 70.468 & 4,55 & $\begin{array}{l}8.741 \\
(-43,53 \%)\end{array}$ & $\begin{array}{l}48.684 \\
(-30,91 \%)\end{array}$ & 5,57 \\
\hline
\end{tabular}

Fuente: Elaboración propia a partir de los datos del Ministerio de Empleo y Seguridad Social. Disponible en: http://www.empleo.gob.es/es/sec trabajo/autonomos/economiasoc/EconomiaSocial/estadisticas/index.htm. Fecha de consulta: 20 de enero de 2018.

En conclusión:

- La sociedad de responsabilidad limitada laboral deja de ser una figura atractiva para acoger a los nuevos proyectos empresariales.

- Hay una mortandad y/o pérdida de condición laboral superior a la de su figura jurídica de base. 
Ambos hechos se explican, al menos en parte, por rigideces en la norma que las regulaba, la Ley 4/1997, de 24 de marzo, de sociedades laborales.

Es en este contexto en el que se promulga la nueva Ley 44/2015, de 14 de octubre, de Sociedades Laborales y Participadas.

\section{Aportaciones de la Ley 44/2015, de 14 de octubre, de Sociedades Laborales y Participadas}

Como se ha referido en el epígrafe precedente, en el período de crisis económica en España, las sociedades de responsabilidad limitada no laborales han mantenido su ritmo de creación al servir de vehículo de creación de empresas a proyectos empresariales de muy reducido tamaño, en muchos casos con solo dos socios (también trabajadores en la mayor parte de los casos). En este contexto, era previsible que la dimensión media de las sociedades de responsabilidad limitada laborales se hubiera reducido y ha ocurrido lo contrario. Esto se justifica, en gran medida, por la limitación establecida en el artículo 5.3 de la Ley 4/1997, de 24 de marzo, de sociedades laborales, que establece que ningún socio puede tener más de un tercio del capital social, hecho que obligaba a que las sociedades laborales comenzaran su actividad con al menos tres socios.

La actualización de este requisito en la nueva redacción legal que permite que "la sociedad laboral se constituya inicialmente por dos socios trabajadores con contrato por tiempo indefinido, en la que tanto el capital social como los derechos de voto estarán distribuidos al cincuenta por ciento, con la obligación de que en el plazo máximo de 36 meses se ajusten al límite establecido en este apartado" (art. 1.2.b) contribuirá, sin duda, a que muchas nuevas empresas puedan considerar la posibilidad de ser laborales.

Pero el alarmante descenso en el número de sociedades laborales activas no se justifica solo por su menor ritmo de creación, sino también, en gran medida, por un llamativo número de bajas societarias.

A priori, no hay motivo para pensar que las sociedades de responsabilidad limitada laborales tuvieran que tener mayores problemas para afrontar la situación de crisis económica que las entidades que conforman su base societaria de referencia, las sociedades de responsabilidad limitada. Si acaso, tendría que ser, al contrario. El hecho de que no haya sido así, sugiere que un importante porcentaje de las bajas societarias en el caso de las sociedades laborales (fundamentalmente sociedades de responsabilidad limitada) son empresas que han perdido su calificación laboral (por diversas causas) pero han continuado su actividad.

Probablemente el motivo fundamental de lo anterior tiene que ver con lo establecido en el artículo 1.2. de la Ley 4/1997 que regulaba el número de horasaño trabajadas por los trabajadores contratados por tiempo indefinido que no sean socios. La nueva Ley de sociedades laborales y participadas, en su artículo 1.2.c) da un paso importante para evitar nuevas pérdidas de calificación al establecer "que el número de horas-año trabajadas por los trabajadores contratados por tiempo indefinido que no sean socios no sea superior al cuarenta y nueve por ciento del cómputo global de horas-año trabajadas en la sociedad laboral por el conjunto de los socios trabajadores". 


\section{Sociedad laboral calificada frente a sociedad laboral calificable}

Con la actual redacción legal, muchas de las sociedades laborales que han perdido su calificación en este periodo de crisis económica podrían haber mantenido su condición laboral. Pasaron de ser sociedades laborales con reconocimiento jurídico a sociedades laborales "de hecho".

Actualmente podrían volver a solicitar su calificación laboral, pero probablemente muchas no lo harán al no encontrar suficientes estímulos para ello. Continuarán siendo, eso sí, sociedades laborales "de hecho", "calificables", como otras muchas sociedades anónimas y, sobre todo, sociedades de responsabilidad limitada que, cumpliendo con los requisitos legales actuales no solicitan ser laborales bien por desconocimiento de esta posibilidad o bien porque no encuentran suficiente motivación para ello.

La realidad de las sociedades laborales "de hecho", "calificables", su cuantificación, es determinante para conocer el peso de la participación de los trabajadores en la empresa española.

Así, se considera sociedad laboral "de hecho" (Bel, et al, 2015) aquella sociedad anónima o de responsabilidad limitada que cumple los requisitos establecidos en el artículo 1.2 de la Ley. En concreto:

1. Que al menos la mayoría del capital social sea propiedad de trabajadores que presten en ellas servicios retribuidos de forma personal y directa, en virtud de una relación laboral por tiempo indefinido (a efectos de este trabajo, en adelante, será considerado requisito 1).

2. Que ninguno de los socios sea titular de acciones o participaciones sociales que representen más de la tercera parte del capital social, salvo que la sociedad laboral se constituya inicialmente por dos socios trabajadores con contrato por tiempo indefinido, en la que tanto el capital social como los derechos de voto estarán distribuidos al cincuenta por ciento, con la obligación de que en el plazo máximo de 36 meses se ajusten al límite establecido en este apartado o se trate de socios que sean entidades públicas, de participación mayoritariamente pública, entidades no lucrativas o de la economía social, en cuyo caso la participación podrá superar dicho límite, sin alcanzar el cincuenta por ciento del capital social (requisito 2).

3. Que el número de horas-año trabajadas por los trabajadores contratados por tiempo indefinido que no sean socios no sea superior al cuarenta y nueve por ciento del cómputo global de horas-año trabajadas en la sociedad laboral por el conjunto de los socios trabajadores (requisito 3).

Este concepto se integraría, por tanto, de todas aquellas sociedades que reúnen requisitos básicos (los referidos arriba) para optar a la consideración jurídica de sociedad laboral, con independencia de que solicite o no su calificación.

Pero no todas las sociedades laborales calificables son calificadas. El artículo 1 de la Ley 44/2015, de 14 de octubre, de Sociedades Laborales y Participadas, en su apartado 1 dice que "las sociedades laborales son aquellas sociedades anónimas o de responsabilidad limitada que se someten a los preceptos establecidos en la presente ley". 
Por su parte, el artículo 2, en su apartado 2 establece: "la calificación de «Sociedad Laboral» se otorgará previa solicitud de la sociedad, a la que acompañará la documentación que se determine reglamentariamente”.

De lo anterior se deriva que el reconocimiento jurídico de sociedad laboral pasa necesariamente por la solicitud expresa de la sociedad para tener tal condición. A efectos de este estudio, todas las sociedades laborales con reconocimiento jurídico tienen la consideración de sociedades laborales "calificadas".

En consecuencia, las sociedades laborales "calificadas" son un subconjunto de las sociedades laborales ·calificables" o "de hecho".

La inclusión del concepto de sociedad participada en la ley permite reconocer la realidad de las sociedades laborales "no calificadas" dado que, como se ha dicho en un epígrafe precedente, entendemos que la intención del legislador al contemplar esta nueva figura ha sido regular toda forma de participación de los trabajadores en la empresa.

En consecuencia, las sociedades laborales "no calificadas" tendrán la consideración de sociedades participadas.

De este modo, la participación de los trabajadores en las sociedades de capital en España quedaría claramente delimitada por el conjunto de las sociedades laborales "de hecho" o calificables (adición de las "calificadas" y "no calificadas") y otras sociedades participadas.

\section{Una aproximación cuantitativa de las sociedades participadas}

El objetivo básico del proyecto en que se basa este trabajo ha sido buscar una aproximación cuantitativa de las sociedades participadas que se crean y que hay en España a partir del concepto de sociedad laboral "calificable", partiendo de un trabajo de campo realizado en gran parte del territorio español sobre sociedades de responsabilidad limitada activas y de nueva creación.

El estudio, básicamente, ha supuesto contrastar las siguientes hipótesis que parten de la observación de la realidad, del análisis estadístico de la evolución de las diferentes formas jurídicas de empresa y de la revisión de estudios teóricos que ponen de manifiesto el carácter participativo de las microempresas:

- Hipótesis 1.

Un porcentaje muy elevado de las microempresas constituidas en España bajo la forma jurídica de sociedades de responsabilidad limitada, son, de hecho, sociedades laborales.

La sociedad de responsabilidad limitada laboral es la forma societaria natural de puesta en marcha de proyectos empresariales no unipersonales, dado que lo habitual en nuestro país es que se creen nanoempresas con forma de sociedad de responsabilidad limitada en la que todos los socios también trabajan en la empresa $\mathrm{y}$, además, controlan democráticamente la toma de decisiones (el capital suele repartirse por igual).

Detrás de los motivos por los que esta realidad no se concreta en sociedades laborales reconocidas jurídicamente se encuentra el que sus socios trabajadores o 
no conocen la posibilidad de acceder a la condición laboral o no encuentran suficiente motivación para solicitar la calificación.

- Hipótesis 2.

Un porcentaje muy elevado de las sociedades de responsabilidad limitada activas reúnen los requisitos legales para tener la consideración laboral.

Los motivos, en este caso, son los mismos que los considerados en el apartado anterior.

- Hipótesis 3.

Un porcentaje muy elevado de las sociedades de responsabilidad limitada laborales que han perdido su condición jurídica en los últimos años cumplirían los requisitos actuales para tener la consideración laboral.

Esta hipótesis se apoya en la actual redacción del artículo 1.2. de la Ley 44/2015, de 14 de octubre, de Sociedades Laborales y Participadas.

En definitiva, las hipótesis anteriores son las que soportan la existencia de las sociedades laborales "no calificadas" (Tabla 5)

Tabla. 5. Motivos que justifican la existencia de sociedades laborales "no calificadas"

Motivo por el que no se tiene el reconocimiento jurídico

Causa que lo provoca

\begin{tabular}{|c|l|}
\hline Pérdida de condición laboral & $\begin{array}{l}\text { Incumplimiento de requisitos } \\
\text { establecidos por la Ley 4/1997 }\end{array}$ \\
\hline No solicitud de calificación & $\begin{array}{l}\text { Desconocimiento de la figura de } \\
\text { sociedad laboral } \\
\text { Falta de atractivos para solicitar la } \\
\text { calificación }\end{array}$ \\
\hline
\end{tabular}

Fuente: Elaboración propia

La metodología seguida en este estudio se basa en un trabajo de campo mediante el cual se ha solicitado la cumplimentación de un cuestionario a dos colectivos:

- $\quad$ Sociedades de responsabilidad limitada activas a fecha 30.06.2016 con independencia de su año de creación.

- Sociedades de responsabilidad limitada activas a fecha 30.06.2016 creadas en los años 2015-2016.

El contenido del cuestionario básicamente ha contemplado las siguientes variables:

- Número de socios

- $\quad$ Número de trabajadores 
- Número de trabajadores por tiempo indefinido que son socios ¿horas/año?

- $\quad$ Porcentaje del capital en poder de trabajadores por tiempo indefinido

- $\quad$ Peso en el capital social del socio que más capital posea

- $\quad$ Peso en el capital social del socio-trabajador que más capital posea

- ¿Saben de la existencia de la Ley 44/2015 de 14 de octubre, de Sociedades Laborales y Participadas?

- Si conocen la posibilidad de haber sido Sociedad Laboral ¿Por qué no lo consideraron (en su caso)?

- ¿Se consideran una sociedad participada? (se les enseña el artículo 19 de la Ley)

Se han elegido las siguientes Comunidades Autónomas sobre la base de que se estima que representan adecuadamente lo que ocurre en el conjunto de territorio español:

$\begin{array}{ll}- & \text { Asturias } \\ - & \text { Castilla-León } \\ - & \text { Galicia } \\ - & \text { Madrid } \\ - & \text { Murcia } \\ - & \text { Navarra } \\ - & \text { País Vasco }\end{array}$

Para la parte del estudio que pretende identificar qué porcentaje de sociedades de responsabilidad limitada activas estarían en condiciones de solicitar su calificación como sociedades laborales, se han identificado de la base de datos ORBIS, a través de una búsqueda booleana, empresas con actividad atendiendo a los siguientes criterios:

- $\quad$ Localización: Las diferentes Comunidades Autónomas referidas.

- $\quad$ Forma jurídica: Sociedades de responsabilidad limitada.

- $\quad$ Número de empleados: Entre 0 y 9.

Se han excluido empresas que no disponen de datos financieros.

Por su parte, para el estudio que pretende identificar qué porcentaje de sociedades de responsabilidad limitada de nueva creación estarían en condiciones de solicitar su calificación como sociedades laborales, de la base de datos ORBIS se han identificado, por el mismo procedimiento que en el caso anterior, empresas con actividad atendiendo a los siguientes criterios:

- $\quad$ Localización: Las diferentes Comunidades Autónomas referidas.

- $\quad$ Forma jurídica: Sociedades de responsabilidad limitada.

- $\quad$ Año de comienzo de actividad: Desde 2015.

- $\quad$ Número de empleados: Entre 0 y 9.

Al igual que en el otro estudio, se han excluido empresas que no disponen de datos financieros.

El nivel de profundidad de los dos estudios planteados se ha concebido distinto habida cuenta de la gran diferencia en la población que requeriría, en el caso del 
estudio de sociedades con actividad, un análisis en mayor profundidad para que la muestra fuera totalmente representativa.

Así las cosas, se ha planteado el estudio de sociedades de nueva creación pretendiendo la máxima representatividad posible de la muestra y, de este modo, poder extraer conclusiones válidas incluso desagregando por Comunidades Autónomas. En el estudio de sociedades activas, sin embargo, se pretende una primera aproximación a la realidad objeto de investigación y es por ello por lo que los resultados que se ofrecen son referidos para el conjunto de la muestra, esto es, como valor agregado del resultado obtenido para las ocho Comunidades Autónomas analizadas que se considera representativo del conjunto nacional.

A este fin, se ha determinado un tamaño muestral del 10 por ciento de las nuevas empresas creadas con forma de sociedad de responsabilidad limitada en el año 2015 según la base de datos de referencia (ORBIS, que se basa en la información de SABI) y un 0,1 por ciento de las entidades activas.

El proceso de encuestación ha sido desarrollado por la Confederación Empresarial de Sociedades Laborales (CONFESAL) apoyado por las organizaciones territoriales que han sido seleccionadas para el estudio y los resultados obtenidos han superado la muestra prevista inicialmente, llegando al 10,29 por ciento en el caso de las empresas de nueva creación.

\section{Resultados}

\subsection{Valoración de las causas por las que las sociedades de nueva creación no acceden a la condición laboral}

Como era previsible, un porcentaje muy significativo de sociedades de responsabilidad limitada de nueva creación no podrían ser nunca laborales por tratarse de sociedades unipersonales, pero además, en el análisis del apartado de observaciones contempladas en el cuestionario se evidencia la existencia de muchos negocios familiares en los que el capital se reparte entre dos o tres socios a lo sumo siendo solo uno (o dos) de ellos los que trabajan en la sociedad. El reparto de capital no suele ser igualitario, sino que la mayor parte (a veces hasta el 95 por ciento) se encuentra en manos del socio trabajador.

Podrían ser sociedades unipersonales pero deciden incorporar como socios a familiares en condición exclusivamente de capitalistas (algunos manifiestan expresamente que buscaban evitar tener la consideración de sociedad limitada unipersonal). En algunos de estos casos se da la circunstancia adicional de que nacen con trabajadores no socios (muchos más que socios trabajadores, si bien algunos tienen contrato a tiempo parcial).

A pesar de no haberse cuantificado concretamente, los numerosos casos en los que se expresa en el cuestionario nos hacen pensar que se trata del colectivo mayoritario entre las no calificables que no son unipersonales. Así las cosas, hay básicamente cuatro tipos de sociedades de responsabilidad limitada microempresas no calificables, siendo las dos primeras categorías las más habituales:

1. Sociedades unipersonales. 
2. Sociedades familiares en las que solo hay un socio trabajador y un socio capitalista.

a. Con mayoría del capital en poder del socio trabajador (incumpliendo requisito 2): lo más común.

i. Con trabajadores no socios (incumpliendo adicionalmente el requisito 3, salvo cuando los trabajadores son a tiempo parcial).

ii. Sin trabajadores no socios.

b. Con el capital distribuido por igual entre los dos socios (incumpliendo requisito 1).

i. Con trabajadores no socios (incumpliendo adicionalmente el requisito 3, salvo cuando los trabajadores son a tiempo parcial).

ii. Sin trabajadores no socios.

3. Sociedades familiares en las que hay dos socios trabajadores, pero también uno o más socios capitalistas.

a. Con mayoría del capital en poder de los socios trabajadores a partes iguales (cumplen mayoritariamente el requisito 2), siendo la aportación del socio o socios capitalistas minoritaria.

i. Con trabajadores no socios (incumpliendo habitualmente el requisito 3, salvo cuando los trabajadores son a tiempo parcial).

ii. Sin trabajadores no socios.

b. Con el capital distribuido por igual entre todos los socios (lo que a veces provoca incumpliendo requisito 1 ).

i. Con trabajadores no socios (incumpliendo adicionalmente el requisito 3 , salvo cuando los trabajadores son a tiempo parcial).

ii. Sin trabajadores no socios.

4. Otras, derivadas del incumplimiento de alguno de los requisitos, pero con causas muy diversas que no permiten categorización (por ejemplo, actividades estacionales como socorristas, que suelen tener muchos contratados e incumplen requisito 3 ).

La nueva Ley de Sociedades Laborales y Participadas contribuye, sin duda, a ampliar el colectivo de sociedades calificables.

La actualización del requisito 2 permite, tal y como se desprende del análisis realizado, que un porcentaje muy importante de nuevas sociedades de responsabilidad limitada de reducido tamaño puedan cumplir con el mismo para solicitar la calificación laboral y la relajación del requisito 3 va a provocar evitar nuevas pérdidas de calificación y, aun siendo el de más difícil cumplimento, va a facilitar que nuevas iniciativas puedan tener la condición laboral.

\subsection{Contraste de hipótesis 1}

Esta hipótesis se verifica dado que el 16,20 por ciento de las sociedades de responsabilidad limitada de nueva creación con menos de 10 trabajadores cumplen con los tres requisitos para optar a la calificación laboral, lo cual supone que podrían optar a la calificación laboral.

Si solo se tuvieran en cuenta las sociedades de responsabilidad limitada de nueva constitución en las que al menos hubiera dos socios (esto es, proyectos no 
unipersonales), el porcentaje anterior ascendería al 24,37 por ciento, lo cual no hace sino reafirmar la hipótesis planteada.

De las respuestas obtenidas se desprende que, efectivamente, los principales motivos que provocan el distanciamiento entre sociedades calificadas y calificables son la falta de información sobre la posibilidad de concretarse los proyectos en sociedades laborales por parte de los promotores (dado que los prescriptores no lo transmiten) y la falta de motivaciones suficientes para solicitar la calificación (que es también un argumento en poder de prescriptores para no recomendar la calificación).

\subsection{Contraste de hipótesis 2}

De la muestra de sociedades activas el 7,30 por ciento de las sociedades de responsabilidad limitada con actividad, con menos de 10 trabajadores, podrían optar a la calificación laboral.

Aunque el porcentaje de sociedades calificables en entidades de nueva creación es más del doble (16,20 por ciento), se considera que este porcentaje es suficientemente elevado y acorde con lo esperado justificándose la diferencia por dos argumentos:

Con el paso del tiempo las empresas crecen y la contratación de trabajadores no socios, así como la entrada de nuevos socios, provoca el incumplimiento de requisitos.

- Pasados los 36 primeros meses, el requisito 2 se hace más exigente obligando a la entrada de un nuevo socio. La exigencia de capital social máximo en poder de un socio pasa a ser de un tercio, lo que hace que haya un porcentaje menor de entidades que cumplen con esta condición.

\subsection{Contraste de hipótesis 3}

Esta hipótesis se soporta en la actual redacción del artículo 1.2. de la Ley 44/2015, de 14 de octubre, de Sociedades Laborales y Participadas".

Pues bien, del estudio realizado se desprende que del total de sociedades activas existentes antes de la entrada en vigor de la Ley 44/2015, un 5,5 por ciento perdieron su condición laboral por el incumplimiento de los límites establecidos por la Ley entonces vigente que podrían haber mantenido su condición en los términos en que está redactada la Ley actual, porcentaje que se considera suficientemente significativo como para dar por válida la hipótesis planteada.

\section{Conclusiones}

El estudio realizado ha permitido realizar las siguientes estimaciones:

1. Estimación de la cifra de sociedades de responsabilidad limitada laborales "de hecho", "calificables" activas en nuestro país.

Del estudio de sociedades activas se desprende que el 7,30 por ciento de las sociedades de responsabilidad limitada activas con menos de 10 trabajadores podrían optar a la calificación laboral, esto es, serían sociedades calificables. En 
consecuencia, del total de sociedades de responsabilidad limitada microempresas activas a 31 de diciembre de 2016, 77.468 serían calificables. Esta cifra ascendería hasta las 84.111 si el porcentaje anterior lo diéramos por válido para sociedades de 10 o más trabajadores.

2. Estimación de la cifra de sociedades de responsabilidad limitada laborales "de hecho", "calificables" creadas en los últimos 2 años.

Del estudio de sociedades de nueva creación se concluye que el 16,20 por ciento de las sociedades de responsabilidad limitada de nueva creación con menos de 10 trabajadores podrían optar a la calificación laboral, esto es, serían sociedades calificables. Así las cosas, del total de sociedades de responsabilidad limitada microempresas constituidas en 2016, 15.712 serían calificables. Esta cifra ascendería hasta las 16.133 si el porcentaje anterior lo diéramos por válido para sociedades de 10 o más trabajadores.

Si tomáramos como referencia el año 2015 , los datos no serían muy diferentes habida cuenta de que el número de sociedades de responsabilidad limitada creadas fue muy similar. De acuerdo con la misma fuente, en este año se constituyeron 90.460 sociedades de responsabilidad limitada, 87.860 de menos de 10 trabajadores y 2.604 de 10 o más.

En consecuencia, podemos concluir que del total de sociedades de responsabilidad limitada microempresas constituidas en 2015, 14.234 serían calificables. Esta cifra ascendería hasta las 14.656 si el porcentaje anterior lo diéramos por válido para sociedades de 10 o más trabajadores.

En definitiva, en el periodo 2015-2016 se han constituido en nuestro país cerca de 30.000 sociedades de responsabilidad limitada calificables.

3. Estimación de la cifra de sociedades de responsabilidad limitada laborales que han perdido su condición jurídica en los últimos años pero que pueden ser consideradas "de hecho", nuevamente calificadas, por tanto.

El 29,18 por ciento de las sociedades de responsabilidad limitada microempresas activas cumplen con el requisito de limitación de trabajadores indefinidos no socios con relación al total de socios trabajadores según la actual redacción legal. Si se tomara como referencia la Ley de 1997, cumplirían con el requisito un porcentaje sensiblemente inferior, el 23,61 por ciento del total. En consecuencia, un 5,58 por ciento de empresas pueden cumplir este requisito actualmente pero no lo cumplían hasta la promulgación de la nueva Ley. En otros términos, y trasladándolo a las sociedades laborales que, en definitiva, son sociedades calificables, del total de sociedades activas existentes antes de la entrada en vigor de la Ley 44/2015, alrededor de un 5,5 por ciento perdieron su condición laboral por el incumplimiento de los límites establecidos por la Ley entonces vigente que podrían haber mantenido su condición en los términos en que está redactada la Ley actual.

4. Estimación de la cifra de sociedades participadas activas con forma de sociedad de responsabilidad limitada en nuestro país y su peso con relación al conjunto. 
Sobre la base de la información disponible en el DIRCE y a partir del dato obtenido en el estudio que indica que el 39,48 por ciento de las empresas encuestadas son participadas, podemos concluir que, del total de sociedades de responsabilidad limitada microempresas activas a 31 de diciembre de 2016, 419.240 serían participadas. Esta cifra ascendería hasta las 455.188 si el porcentaje anterior lo diéramos por válido para sociedades de 10 o más trabajadores.

5. Estimación de la cifra de sociedades participadas de nueva creación con forma de sociedad de responsabilidad limitada en nuestro país y su peso con relación al conjunto.

El 31,28 por ciento de las sociedades objeto de estudio tiene la consideración de participadas, lo que significa que, del total de sociedades de responsabilidad limitada microempresas constituidas en 2016, 30.340 serían participadas. Esta cifra ascendería hasta las 31.151 si el porcentaje anterior lo diéramos por válido para sociedades de 10 o más trabajadores.

Tomando como referencia el año 2015 , serían participadas 27.487 sociedades de responsabilidad limitada microempresas del total creadas ese año, cifra que aumentaría hasta las 28.302 entidades si se incluyeran sociedades de 10 o más trabajadores.

En definitiva, en los años 2015-2016 se han constituido en España por encima de 57.000 sociedades de responsabilidad limitada participadas.

En definitiva, la nueva Ley de Sociedades Laborales y Participadas contribuye, sin duda, a ampliar el colectivo de sociedades calificables, con la posibilidad de crear sociedades laborales con solo dos socios (trabajadores) y mantener su condición sin alterar la composición en el capital hasta los 36 meses de inicio de actividad y con la ampliación del límite de trabajadores indefinidos no socios con relación al colectivo de socios trabajadores.

Pero lo anterior no es garantía de que la brecha entre sociedades calificables y calificadas disminuya. Puede ocurrir, de hecho, todo lo contrario.

El trabajo de campo realizado pone en evidencia el hecho de que podrían calificarse anualmente que más de 15.000 sociedades de responsabilidad limitada, cuando en realidad solicitan la calificación poco más de 400 (en torno al 2,6 por ciento).

Son dos, los motivos que se consideran esenciales para la no calificación de sociedades que pueden optar a ello:

- $\quad$ El insuficiente conocimiento de la sociedad laboral.

- La falta de atractivos para solicitar su calificación (una fiscalidad muy poco atractiva en términos relativos y la inexistencia de medidas de política activa de empleo exclusivas dirigidas a sociedades en las que se priorice la condición laboral: como hasta hace poco tiempo era el pago único de la prestación por desempleo, a lo que podría añadirse otros aspectos relacionados con la adscripción a los regímenes de la Seguridad Social).

Como ya se ha mencionado, ambos motivos son determinantes en la falta de atención que presta a esta figura un colectivo que se considera esencial en el hecho de la decisión última de la forma jurídica elegida para realizar una actividad empresarial: los asesores o prescriptores empresariales. 
El escaso, a todas luces insuficiente, conocimiento de la figura de la sociedad laboral pasa por realizar campañas informativas y formativas, particularmente entre quienes asesoran proyectos emprendedores y entre jóvenes en las diferentes etapas del modelo educativo.

La falta de incentivos suficientes para solicitar la calificación laboral es el asunto más complejo. Sociedades cooperativas y sociedades laborales han sido fórmulas promovidas, conocidas y recomendadas por prescriptores durante el tiempo en el que el acceso al pago único de la prestación por desempleo para quienes querían constituir sociedades podía hacerse solo a través de este tipo de empresas. La pérdida de esta ventaja relativa, unida a una fiscalidad insuficientemente atractiva y una falta de bonificación y promoción en materia de Seguridad Social comparada con la ofrecida a otros instrumentos jurídicos de promoción de proyectos empresariales, como las sociedades civiles, es detonante de la baja calificación. Por tanto, la posibilidad de articular una fiscalidad diferenciada se considera capital para que el colectivo de sociedades laborales calificadas y calificables se aproxime cuantitativamente.

\section{Referencias bibliográficas}

Alguacil Marí, M. P. (2010) La tributación de las empresas de participación de los trabajadores (Cooperativas de Trabajo y Sociedades Laborales): apuntes para una reforma. REVESCO. Revista de Estudios Cooperativos, $\mathrm{n}^{\mathrm{o}}$ 102, pp. 24-53.

Amos and Martins (2002) Privatization in Portugal: employee owners or just happy employees?. Coimbra: Grupo de Estudos Monetários e Financeiros (GEMF).

Andreu Martí, M. M. (2010) Luces y sombras de la reforma de la Ley de Sociedades Laborales. Ciriec- España. Revista Jurídica de Economía Social y Cooperativa, $\mathrm{n}^{\mathrm{o}} 21$, pp. 119-144.

Bahia Machado, R. (2011) Participación de los trabajadores en el capital de la empresa: su implementación y viabilidad en Portugal: análisis de 14 paises de la Unión Europea. Tesis Doctoral. Madrid: Universidad Complutense de Madrid. ISBN: 978-84-695-03218.

Bel Durán, P.; Fernández Guadaño, J.; Lejarriaga Pérez de las Vacas, G. y Martín López, S. (2015) Sociedades laborales de hecho y de derecho en el contexto de la nueva Ley de sociedades laborales y participadas. XXXV Seminario Nacional y XX Seminario Internacional de investigación en materia de organizaciones de participación organizado por la Escuela de Estudios Cooperativos de la UCM, 25 de septiembre en Vigo.

Chaves Ávila, R. y Savall Morera, T. (2013) La insuficiencia de las actuales políticas de Fomento de cooperativas y sociedades laborales frente a la crisis en España. REVESCO. Revista de Estudios Cooperativos, $\mathrm{n}^{\circ}$ 113, pp. 61-91. DOI: 10.5209/rev_REVE.2014.v113.43383.

Cuadrado Serrán, M. y Ciruela Lorenzo, A. M. (2014) Las sociedades cooperativas y las sociedades laborales como motor de desarrollo económico y social. REVESCO. Revista de Estudios Cooperativos, $\mathrm{n}^{\mathrm{0}} 115, \quad$ pp. 57-100. DOI: 10.5209/rev_REVE.2014.v115.45278.

Divar, J. (2015) Noticia de las principales novedades de la nueva Ley de Sociedades Laborales y Participadas. Deusto Estudios Cooperativos, n 7, pp. 13-17. 
EFES (2017) Recent policy decisions regarding employee share ownership in European countries, 11 de enero de 2017. Disponible en: http://www.efesonline.org/INDISPENSABLE/RECENT\%20POLICY\%20DECISIONS \%20REGARDING\%20EMPLOYEE\%20SHARE\%20OWNERSHIP\%20IN\%20EURO PEAN\%20COUNTRIES.pdf.

ESPAÑA. Dictamen del Comité Económico y Social Europeo (CESE), de 21 de octubre de 2010, sobre la participación financiera de los trabajadores en Europa.

ESPAÑA. LEY 4/1997, de 24 de marzo, de Sociedades Laborales. Boletín Oficial del Estado, $\mathrm{n}^{\mathrm{0}} 72$, de 25 de marzo.

ESPAÑA. LEY 44/2015, de 14 de octubre, de Sociedades Laborales y Participadas. Boletín Oficial del Estado, $\mathrm{n}^{\circ} 247$, de 15 de octubre.

EUROPEAN COMMISSION (2014) The Promotion of Employee Ownership and Participation. Final Report.

Fajardo-García, G.; Muñecas, J. y San José, J. (2016) Employee Financial Participation: Spain's Sociedades Laborales. European Parliament: DIRECTORATE GENERAL FOR INTERNAL POLICIES. IP/A/EMPL/2016-01.

Fernández Guadaño, J.; Martín López, S.; Lejarriaga Pérez de las Vacas, G. y Bel Durán, P. (2016) Behavior of Labour Cooperative Societies during the crisis proposals for promotion and consolidation. Ponencia presentada al International Research Conference of the International Co-operative Alliance. Almeria, 24 a 27 de mayo.

Fulton, L. (2015) Worker representation in Europe. Labour Research Department and ETUI. Produced with the assistance of the SEEurope Network. Publicación virtual: http://www.worker-participation.eu/National-Industrial-Relations.

Lejarriaga Pérez de las Vacas, G. y Bel Durán, P. (Directores); Alguacil, P.; Fernández Guadaño, J.; Iturrioz del Campo, J.; López Millán, M.; Martín López, S. y Palomo Zurdo, R. (2017) Delimitación del concepto de "Sociedad Participada": Delimitación conceptual de las diferentes formas en que puede concretarse una sociedad participada sobre la base de las sociedades laborales "calificables". Proyecto de Investigación. Ministerio de Empleo y Seguridad Social.

Lejarriaga Pérez de las Vacas, G. y Bel Durán, P. (directores); Fernández Guadaño, J. y Martín López, S. (2017) Estudio del concepto de "Sociedad Participada" a partir de las sociedades laborales "no calificadas": Una aproximación a su cuantificación a partir del estudio de las sociedades de responsabilidad limitada calificables activas y de nueva creación. Proyecto de Investigación. Confesal y Ministerio de Empleo y Seguridad Social.

Lejarriaga Pérez de las Vacas, G. (2016) La realidad económica de las sociedades laborales en España ante la reforma de la Ley. Ponencia III Jornadas de investigación y docencia en materia de empresas de participación (EEC y AECOOP), 26 febrero.

Lejarriaga Pérez de las Vacas, G.; Fernández Guadaño, J.; Martín López, S. y Bel Durán, P. (2015) Study comparing employee-owned limited liability firms (identified as Sociedades Laborales) "de jure (concerning law) and "de facto" (concerning fact) in Spain”. Ponencia 5th International Research Conference on Social Economy. CiriecPortugal. Lisbon, 15-18 julio.

Lejarriaga Pérez de las Vacas, G.; Bel Durán, P. y Martín López, S. (2016) Propuesta de delimitación conceptual de la participación de los trabajadores en las sociedades de capital en España y aproximación a su cuantificación. Ponencia presentada al XVI Congreso de Investigadores en Economía Social, celebrado en Valencia del 19 al 21 de octubre de 2016. 
Lejarriaga Pérez de las Vacas, G. (2002) Participación financiera de los trabajadores y creación de valor: una propuesta operativa de comportamiento con relación a los objetivos empresariales. Ciriec-España, Revista de Economía Pública, Social y Cooperativa, $\mathrm{n}^{\mathrm{o}}$ 40, pp. 73-98.

Liger, Q.; Stefan, M. y Britton, J. (2016) Social economy. Unión Europea. Disponible en: http://www.europarl.europa.eu/RegData/etudes/STUD/2016/578969/IPOL_STU(2016)5 78969 EN.pdf.

Lowitzsch, J. y Spitsa, N. (2009) Financial participation of employees in the EU-27. Palgrave Macmillan, pp. 85-120. ISBN: 978 0-333-73353-0.

Lowitzsch, J. (2009) Financial participation of employees in the EU-27. Palgrave: Macmillan.

Masa Lorenzo, C.; Iturrioz del Campo, J. y Martín López, S. (2016) Aspectos determinantes del fracaso empresarial: efecto de la proyección social de las sociedades cooperativas frente a otras formas jurídicas. Ciriec-España, Revista de Economía Pública, Social y Cooperativa, $\mathrm{n}^{\mathrm{o}} 88$.

Mathieu, M. (2016) Annual Economic Survey of Employee Share Ownership in European Countries 2015. Brussels: European Federation of Employee Share Ownership.

Vargas Sánchez, A. y Lejarriaga Pérez de las Vacas, G. (Coordinadores) Las empresas de participación en Europa: el reto del siglo XXI (En homenaje al Prof. Dr. Carlos GarcíaGutiérrez Fernández). Madrid: Escuela de Estudios Cooperativos. Madrid, 2002 p. 97.

VVAA. (2007) La tributación de las sociedades laborales. Madrid: Aranzadi.

VVAA. (2008) Reforma de la tributación de las sociedades laborales. Ciriec, Revista jurídica, $\mathrm{n}^{\mathrm{o}} 19$.

Wilke, Mack and Partner (2014) Country reports on Financial Participation in Europe, Prepared for www.worker-participation.eu. Informe publicado en 2007 y actualizado en 2014.

Young, K. y Patel, P. (2016) Employee ownership and firm performance: A variance decomposition analysis of European firms. Journal of Business Research. http://dx.doi.org/10.1016/j.jbusres.2016.08.014. 